\title{
Application of Improved Naive Bayesian-CNN Classification Algorithm in Sandstorm Prediction in Inner Mongolia
}

\author{
Li Tiancheng $(\mathbb{D}$, Ren Qing-dao-er-ji $(\mathbb{D}$, and Qiu Ying \\ School of Information Engineering, Inner Mongolia University of Technology, Hohhot 010051, China \\ Correspondence should be addressed to Ren Qing-dao-er-ji; renqingln@sina.com
}

Received 28 March 2019; Revised 26 June 2019; Accepted 12 August 2019; Published 6 December 2019

Academic Editor: Anthony R. Lupo

Copyright (@) $2019 \mathrm{Li}$ Tiancheng et al. This is an open access article distributed under the Creative Commons Attribution License, which permits unrestricted use, distribution, and reproduction in any medium, provided the original work is properly cited.

\begin{abstract}
Hazards of sandstorm are increasingly recognized and valued by the general public, scientific researchers, and even government decision-making bodies. This paper proposed an efficient sandstorm prediction method that considered both the effect of atmospheric movement and ground factors on sandstorm occurrence, called improved naive Bayesian-CNN classification algorithm (INB-CNN classification algorithm). Firstly, we established a sandstorm prediction model based on the convolutional neural network algorithm, which considered atmospheric movement factors. Convolutional neural network (CNN) is a deep neural network with convolution structure, which can automatically learn features from massive data. Then, we established a sandstorm prediction model based on the Naive Bayesian algorithm, which considered ground factors. Finally, we established a sandstorm prediction model based on the improved naive Bayesian-CNN classification algorithm. Experimental results showed that the prediction accuracy of the sandstorm prediction model based on INB-CNN classification algorithm is higher than that of others and the model can better reflect the law of sandstorm occurrence. This paper used two algorithms, naive Bayesian algorithm and $\mathrm{CNN}$ algorithm, to identify and diagnose the strength of sandstorm in Inner Mongolia and found that combining the two algorithms, INB-CNN classification algorithm had the greatest success in predicting the occurrence of sandstorms.
\end{abstract}

\section{Introduction}

Sandstorms have become an important natural disaster affecting human survival and development. It is of great significance for disaster prevention and mitigation to study the occurrence regularity of sandstorms. Sandstorms often occur in Asia, Africa, Americas, and Australia [1] but have not been reported in Europe. Many studies have been carried out on the climatic characteristics of sandstorms. As the data and methods used are different, the conclusions are also quite different [1].

Chang-qing et al. [2] introduced the sand-dust forecasting model called the Chinese Unified Atmospheric Chemistry Environment for Dust (CUACE/Dust). Their research showed that the results obtained by the CUACE/ Dust model are as good as the finer grids in the key areas of attracting attention. Wei et al. [3] proposed a new location method to detect the location and intensity of sandstorms using remote sensing images. Their research showed that this method can detect the center and intensity of sandstorms quickly and effectively. Han et al. [4] employed an index called surface temperature change (STC) for assisting in sandstorm forecast over Northwest China. Their research showed that the method has little value in predicting sandstorms in Northwest China. According to the national sandstorm data, Guoping et al. [5] found the wind erosion sites in sandstorm-affected areas are all in autumn and with the aggravation of soil erosion, the number of sandstorm days in one year also increases. Lu et al. [6] combined the BP neural network with genetic algorithm to establish and implement a sandstorm prediction model. Their research showed that the genetic neural network method has high accuracy in stability and running speed.

Kim et al. [7] compared two sandstorm models, Asian Dust Aerosol Model (ADAM) and Chinese Unified Atmospheric Chemistry Environment for Dust (CFAS/ Dust). Their research showed that both models produce 
similar results and that the predicted values are in good agreement with the observed results. In and Park [8] proposed a method to simulate such long-distance transmission events. Their research showed that the present model can be used to predict the Huangsha event in Korea. American scholar Akhlaq et al. [9] proposed a hybrid design of a sand and dust storm detection system, which used wireless sensor networks and satellite imaging to detect all types of sand and dust storms. Their research showed that this hybrid method can effectively detect and predict all types of dust storms. Pérez et al. [10] studied the atmospheric model of a sand-dust area. Their research showed that the model can predict the atmospheric life cycle of dust storm erosion.

Nowadays, some scholars have used different methods to analyze and classify satellite cloud images, but relevant studies are few. Remote sensing technology is an important means of mapping coral reef ecosystems. Gholoum et al. [11] classified the images by multiple linear regression analysis. Their research showed that the coral density map drawn by multiple linear regression models is reliable. Texture and shape analysis provides interesting possibilities for describing the structural heterogeneity of classes in high spatial resolution satellite images. Liu et al. [12] proposed an unsupervised ordered classification algorithm. Their research showed that the ordered classification method combined with spectral, texture, and shape information has better classification effect than the traditional method. Campagnolo and Caetano [13] proposed a satellite image classification method based on expert knowledge. The method was applied to real image datasets. Their research showed that the method is more accurate than the classification based on spectral data alone. Land cover classification analysis in satellite images is very important for monitoring ecosystem changes and urban growth over time. Nguyen et al. [14] used deep learning of high-resolution satellite images to generate pixel-based land cover classification.

Throughout the research status of sandstorms, the research scholars mainly had taken the ground climate data of sandstorms as the research object and used data mining algorithms to establish a model, studying the occurrence law of sandstorms. However, there are few studies on the occurrence of sandstorms based on satellite cloud images and convolutional neural network algorithm.

The convolutional neural network algorithm is a kind of deep neural network, which can extract features from massive data. The convolutional neural network algorithm has the features of local connection, downsampling, and weight sharing. But, the sandstorm prediction model based on the convolutional neural network algorithm only considers the influence of atmospheric movement on the occurrence of sandstorms. In order to solve this problem, combining with the naive Bayesian algorithm, we proposed an improved sandstorm prediction algorithm, improved naive Bayesian-CNN classification algorithm (INB-CNN classification algorithm), and established a sandstorm prediction model based on this algorithm and analyzed its prediction accuracy.
Convolutional neural network algorithm and naive Bayesian algorithm are very important data mining algorithms. They are applied to data analysis in the field of meteorology which can explore the internal relations among various meteorological elements and find various potential laws to reveal unknown meteorological theories. They are not only important for meteorological science research but also useful in enriching weather forecasting methods and improving the level of weather forecasting, which plays an active and important role.

\section{Data Sources}

Establishment of a sandstorm prediction model based on the INB-CNN classification algorithm needs three datasets, which are as follows:

China's Series of Strong Dust Storms and Its Supporting Dataset. This dataset comes from the National Meteorological Science Data Sharing Service Platform, which mainly records the occurrence sequence of China's strong dust storms from 1954 to 2007.

Daily Dataset of China's Ground Climate Data. This dataset is derived from the information files of the national monthly reports on the ground. The dataset records the meteorological data of the ground monitoring stations all over the country, such as the local air pressure and temperature.

China Land Regional Cloud Map (IR1) (Data Starting Time: 2005-05-30, 00:00; Data Termination Time: 200909-19, 00:00). This dataset comes from the National Meteorological Science Data Sharing Service Platform.

We used the data from 2005 to 2007 in the three datasets to establish prediction models. The dataset to test the sandstorm prediction model based on the INB-CNN classification algorithm must be synchronized in time.

The daily average of the observed data of four times a day (Beijing Time: 02:00, 08:00, 14:00, and 20:00) obtained from the aforementioned monthly meteorological report data file or real-time database is as follows:

(1) The daily average temperature, relative humidity, and ground temperature are the average values of four time observations. The temperature is $0^{\circ} \mathrm{C}$.

(2) In general, the daily average local pressure and daily average wind speed are the average values of four time observations, but in the absence of self-recording instruments, the daily average air pressure and daily average wind speed are obtained at 08:00, 14:00, and 20:00 time observations from the three time observation stations.

(3) From (1) and (2), the statistical daily average is stipulated.

Therefore, this paper chooses the ground climate data and satellite cloud image data of sandstorm at 02:00, 08:00, 14:00, and 20:00 every day as the test set of the sandstorm prediction model based on the INB-CNN classification algorithm. 


\section{Research on Sandstorm Prediction Model Based on Naive Bayesian Algorithm}

3.1. Data Preprocessing. The data preprocessing is shown in Figure 1.

The ground climate data of sandstorm used in this paper are unevenly distributed under different sandstorm grades. Dataset imbalance will make the prediction model based on this dataset overfit in the level of large amount of data but underfit in the level of small amount of data. According to the feature of the ground climate data of sandstorm, we used the SMOTE algorithm to deal with the problem of data imbalance.

The intensity of the sandstorm is divided into five grades: $1,2,3,4$, and 5 based on the single-station strong sand-dust storm standard. The undersampling method is used to deal with the data of sandstorm grade 1, and the number is reduced to about 4000 lines. The oversampling method, SMOTE algorithm, is used to enhance the data of sandstorm grades $2,3,4$, and 5 , and the number is reduced to about 4000 lines, respectively.

In order to facilitate this study, the experimental dataset is divided into two parts, training set and test set. The proportion is $3: 1$, as shown in Table 1 .

3.2. Establishing Sandstorm Prediction Model Based on Naive Bayesian Algorithm. In order to vividly show the process of establishing a sandstorm prediction model based on the naive Bayesian algorithm, the process is drawn, as shown in Figure 2.

We use the naive Bayesian algorithm to establish the prediction model, where the attributes are independent of each other. For this reason, the "average local pressure," "average temperature," "average relative humidity," "20-20 cumulative precipitation (cumulative precipitation from Beijing Time 20:00 to 08:00)," "small evaporation," "average wind speed," "sunshine hours," and "average temperature" are selected as characteristic attributes from the "daily dataset of surface climate data in China." When the value of an attribute is a continuous variable, it is called a numerical attribute. Usually, we assume that the values of numerical attributes obey normal distribution. The AdaBoost algorithm is used to improve the classification accuracy of the naive Bayesian algorithm. The parameters of the AdaBoost algorithm are set as follows: learning_rate: 0.1 ; base_estimator: GaussianNB (); n_estimators: 50; algorithm: SAMME.R.

\section{Research on Sandstorm Prediction Model Based on Convolutional Neural Network Algorithm}

4.1. Image Annotation. In this paper, infrared satellite cloud images are used, and the format of satellite cloud images used in the experiment is ".AWX." Meteorological mapping software "MeteoInfo" is used to transform satellite cloud image format into ".PNG." Then, according to the standard of single-station strong sand-dust storm, the transformed satellite cloud image is labeled by using the

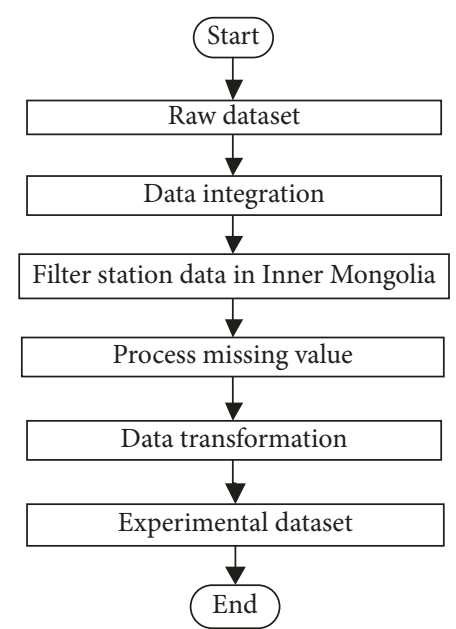

FIGURE 1: Data preprocessing.

TABLE 1: Balanced daily dataset.

\begin{tabular}{lccccccc}
\hline Dataset & 0 & 1 & 2 & 3 & 4 & Total & Unit \\
\hline Training set & 3000 & 3000 & 3000 & 3000 & 3000 & 15000 & Line \\
Test set & 1000 & 1000 & 1000 & 1000 & 1000 & 5000 & Line \\
\hline
\end{tabular}

Python language, and the LMDB data source is made. After that, the satellite cloud image is processed by deep learning framework.

From the dataset of China Series of Strong Dust Storms and its Supporting Dataset, the site data of Inner Mongolia are collected. According the standard of single-station strong sand-dust storm, as shown in Table 2, the sandstorm grade of each station in Inner Mongolia is analyzed. We divided the intensity of sandstorms into five grades: 5 for strong sandstorm, 4 for medium sandstorm, 3 for general sandstorm, 2 for weak sandstorm, and 1 for no sandstorm.

\section{Remarks 1}

(1) In meteorological observation, visibility has been marked by unit length $\mathrm{m}$ since 1980, and previously by levels $0-9$.

(2) When the maximum wind speed is missing, use the average maximum wind speed for ten minutes; when both are missing, only the visibility is used.

(3) Priority should be given to visibility conditions. When the wind speed conditions are not satisfied, this record is also regarded as a reference record and participates in statistics.

At intervals of 30 minutes, the occurrence time of sandstorms at each station is discretized by the hash function, and the maximum intensity of sandstorms at each time point is analyzed. At this time point, the maximum grade of intensity of sandstorm occurrence in Inner Mongolia is used to mark the satellite cloud image at that time.

4.2. Dataset Unbalanced Solution. The amount of infrared satellite cloud image dataset used in this paper varies greatly 


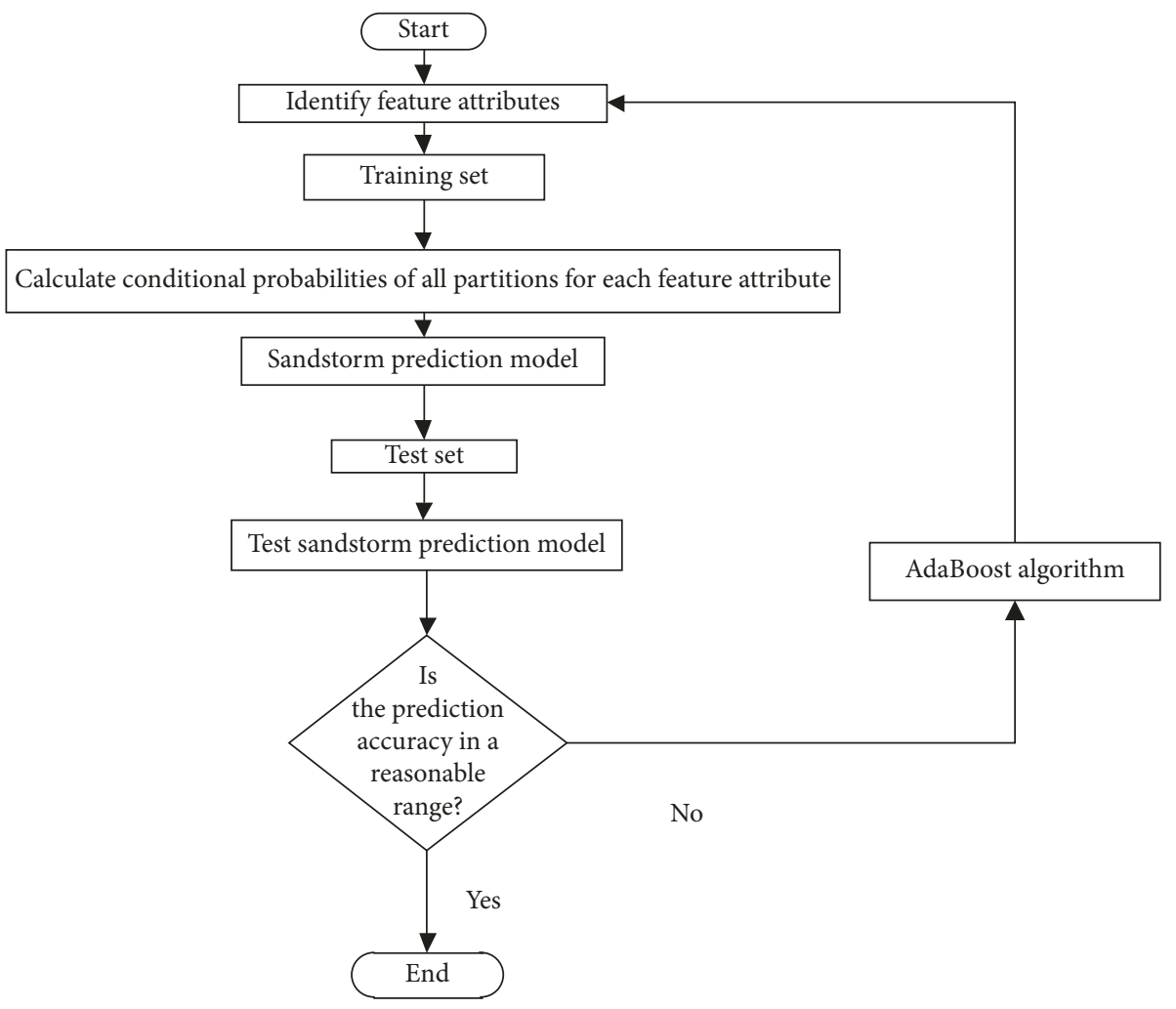

FIGURE 2: Establishment process of sandstorm prediction model based on naive Bayesian algorithm.

TABLE 2: Single-station strong sandstorm standard.

\begin{tabular}{lcc}
\hline Sandstorm intensity & Visibility $V(\mathrm{~m})$ & The maximum wind speed $f(\mathrm{~m} / \mathrm{s})$ \\
\hline Strong & 0 level, 1 level or $V \leq 200$ & $f \geq 20$ \\
Medium & 2 level or $200<V \leq 500$ & $17 \leq f<20$ \\
General & 3 level or $500<V \leq 1000$ & $10 \leq f<17$ \\
\hline
\end{tabular}

in different grades and data imbalance. The unbalanced data will cause the prediction model to overfit at the grade of more data and underfit at the grade of less data. In view of the meteorological feature of sandstorms, we used perspective transformation to enhance satellite cloud images and deal with the problem of data imbalance.

Based on the single-station strong sand-dust storm standard, the intensity of sandstorm occurrence is divided into five grades: $1,2,3,4$, and 5 . In this paper, the undersampling method is used to reduce the number of satellite cloud images labeled 1 to about 4000 pages. At the same time, the perspective transformation is used to enhance the number of satellite cloud images labeled 2, 3, 4, and 5 to about 4000 pages, respectively. The ratio of the number of training set and test set for each grade is divided into $3: 1$.

Sandstorm is a common disastrous weather in spring in northern China. So far, sandstorms in China, and even in the world, are still far from being controlled, and there are still many unknown topics in the field of sandstorm research [15]. It is easy to see that Inner Mongolia in northern China (Figure 3) is an area with high incidence of sandstorms through the transmission route of sandstorm in northern China (Figure 4). Therefore, it is of great significance to study the occurrence regularity of sandstorms in this area.

In this paper, the satellite cloud image dataset of China are taken as the research object, and the sandstorm prediction model is established based on convolutional neural network to study the occurrence law of sandstorms in Inner Mongolia. Taking the infrared satellite cloud image named "FY2C_SEC_IR1_LCN_20060326_1300.png" as an example, the principle of the perspective transformation is illustrated. Firstly, the image is intercepted, and then perspective transformation is performed to rotate the image in space, as shown in Figure 5.

In order to facilitate this study, the number of each category of the experimental dataset is divided into two parts: training set and test set. The ratio is $3: 1$, as shown in Table 3.

4.3. Establishing Sandstorm Prediction Model Based on Convolutional Neural Network Algorithm. Taking the dataset of infrared satellite cloud images as the research object, the convolutional neural network algorithm is used 


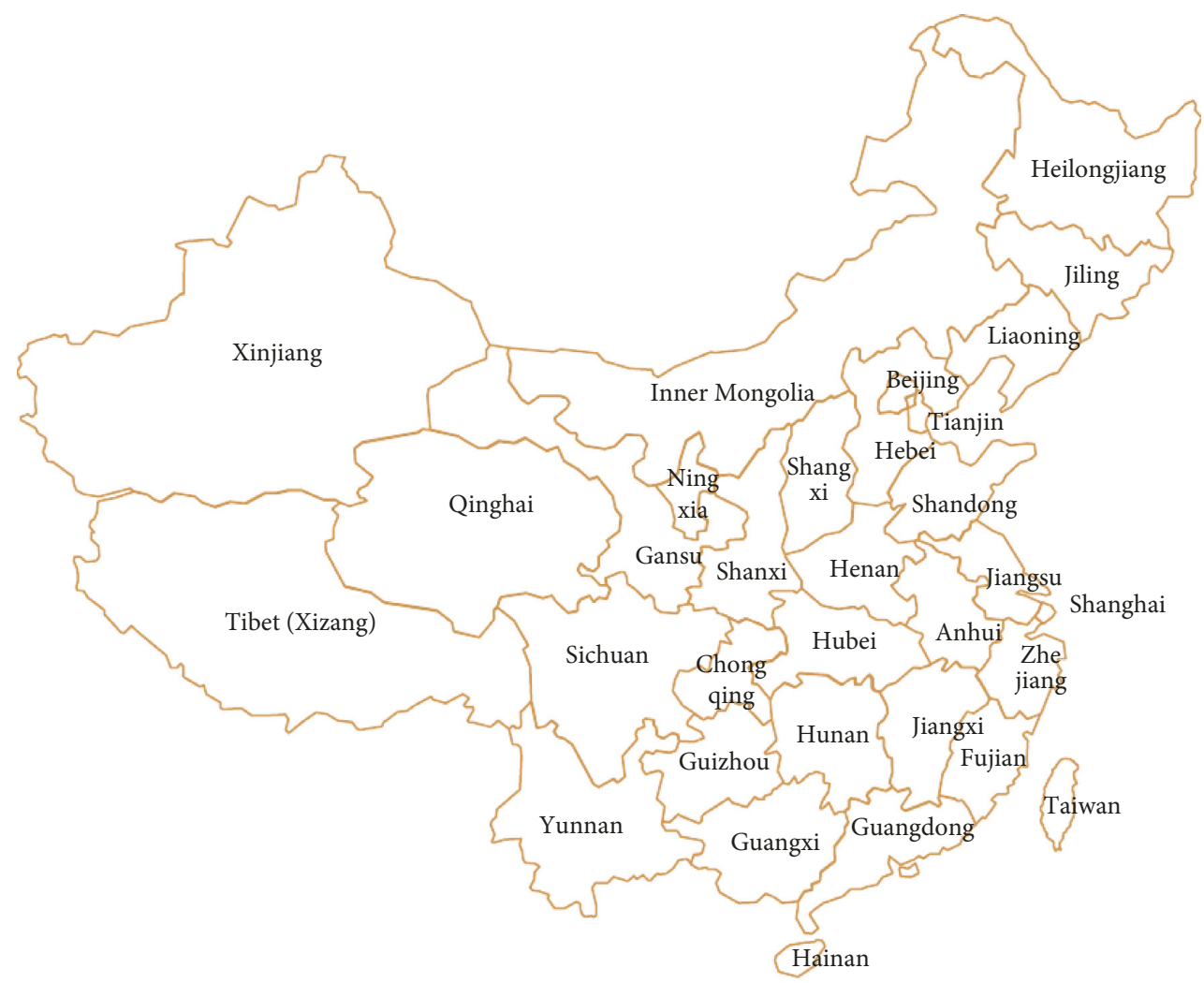

Figure 3: Map of provinces in China.

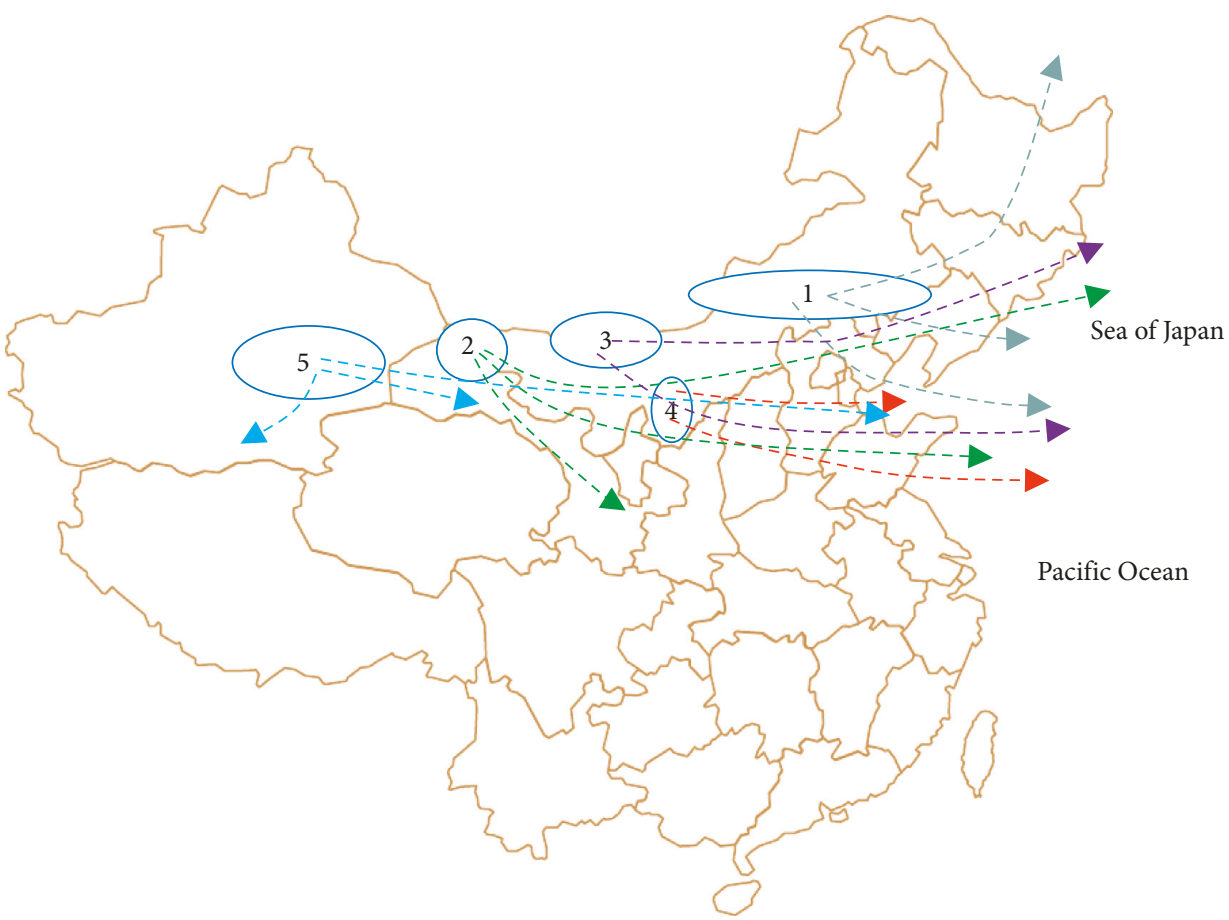

FIGURE 4: Dust sources and transport pathways identified by MODIS [15]. Note: 1 represents sandy lands in central Inner Mongolia and the adjacent area of Mongolia; 2 represents the Gobi Desert in western Inner Mongolia and neighboring areas in Xin Jiang, Gan Su, and southwestern Mongolia; 3 represents the Gobi Desert and sandy deserts of western Inner Mongolia and adjacent areas of southwestern Mongolia; 4 represents sandy lands and deserts along the middle reaches of the Yellow River; 5 represents the rim of the Taklamakan Desert. Colored arrows show the paths of dust transport between Mongolia and northern China, derived from time-series satellite images and the NOAA HYSPLIT model [15]. 


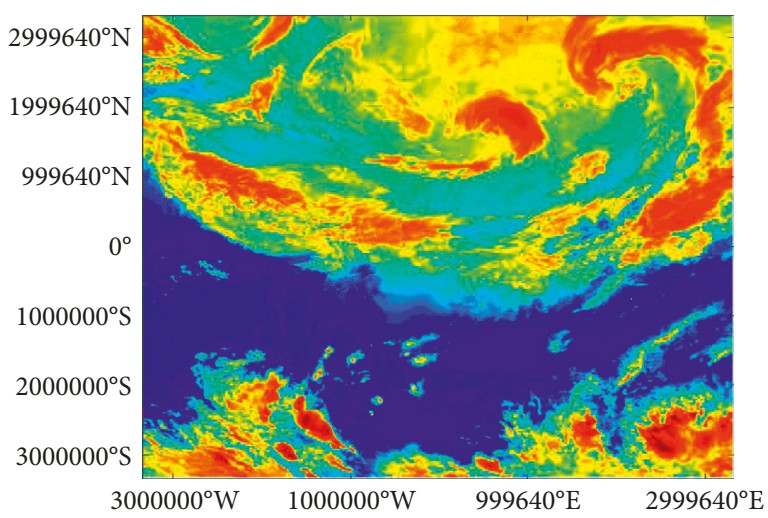

(a)

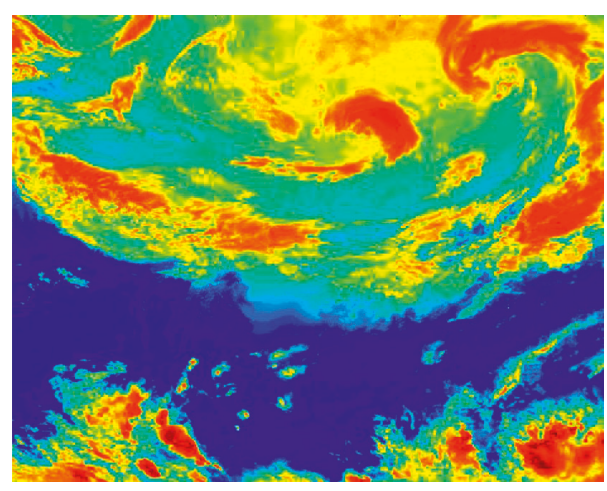

(b)

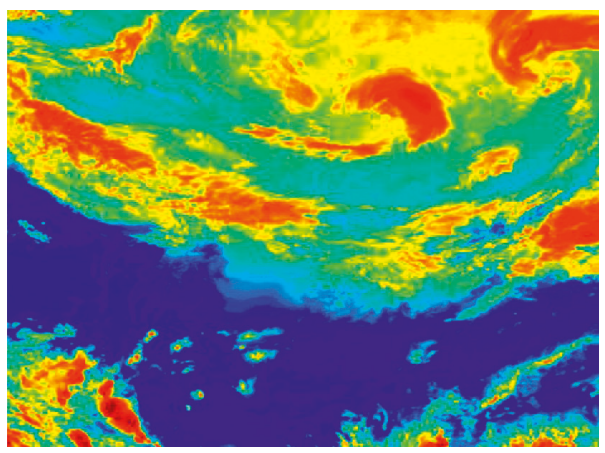

(c)

FIGURE 5: (a) Original cloud image. (b) Postshear satellite cloud image. (c) Transformed satellite cloud image.

TABle 3: Experimental dataset.

\begin{tabular}{lccccccc}
\hline Data set & 0 & 1 & 2 & 3 & 4 & Total & Unit \\
\hline Training set & 3000 & 3000 & 3000 & 3000 & 3000 & 15000 & Page \\
Test set & 1000 & 1000 & 1000 & 1000 & 1000 & 5000 & Page \\
\hline
\end{tabular}

to establish the sandstorm prediction model, and the occurrence law of sandstorm is studied. The process is illustrated in Figure 6.

\section{Research on Sandstorm Prediction Model Based on INB-CNN Classification Algorithm}

5.1. The Principle of Algorithmic Improvement. The occurrence of sandstorm is a natural phenomenon caused by the interaction of atmospheric motion and ground factors. The sandstorm prediction model based on convolutional neural network algorithm considers only the influence of atmospheric motion on the occurrence of sandstorm; the sandstorm prediction model based on the naive Bayesian algorithm considers only the influence of ground factors on the occurrence of sandstorm. In order to study the regularity of sandstorm occurrence and predict the intensity of sandstorm occurrence from a spatial perspective, the INB-CNN classification algorithm is proposed.

The INB-CNN classification algorithm can be applied not only to sandstorm prediction but also to other fields,

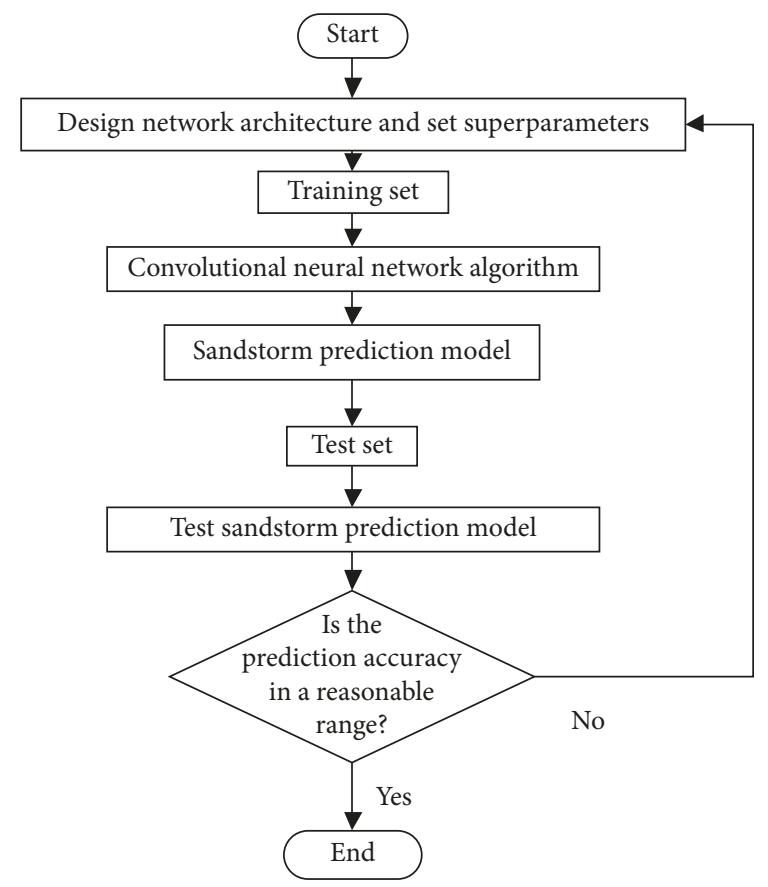

FIGURE 6: Training process of sandstorm prediction model based on convolutional neural network algorithm.

such as image processing and text data processing. The mathematical principle of the INB-CNN classification algorithm is shown in Figure 7. 


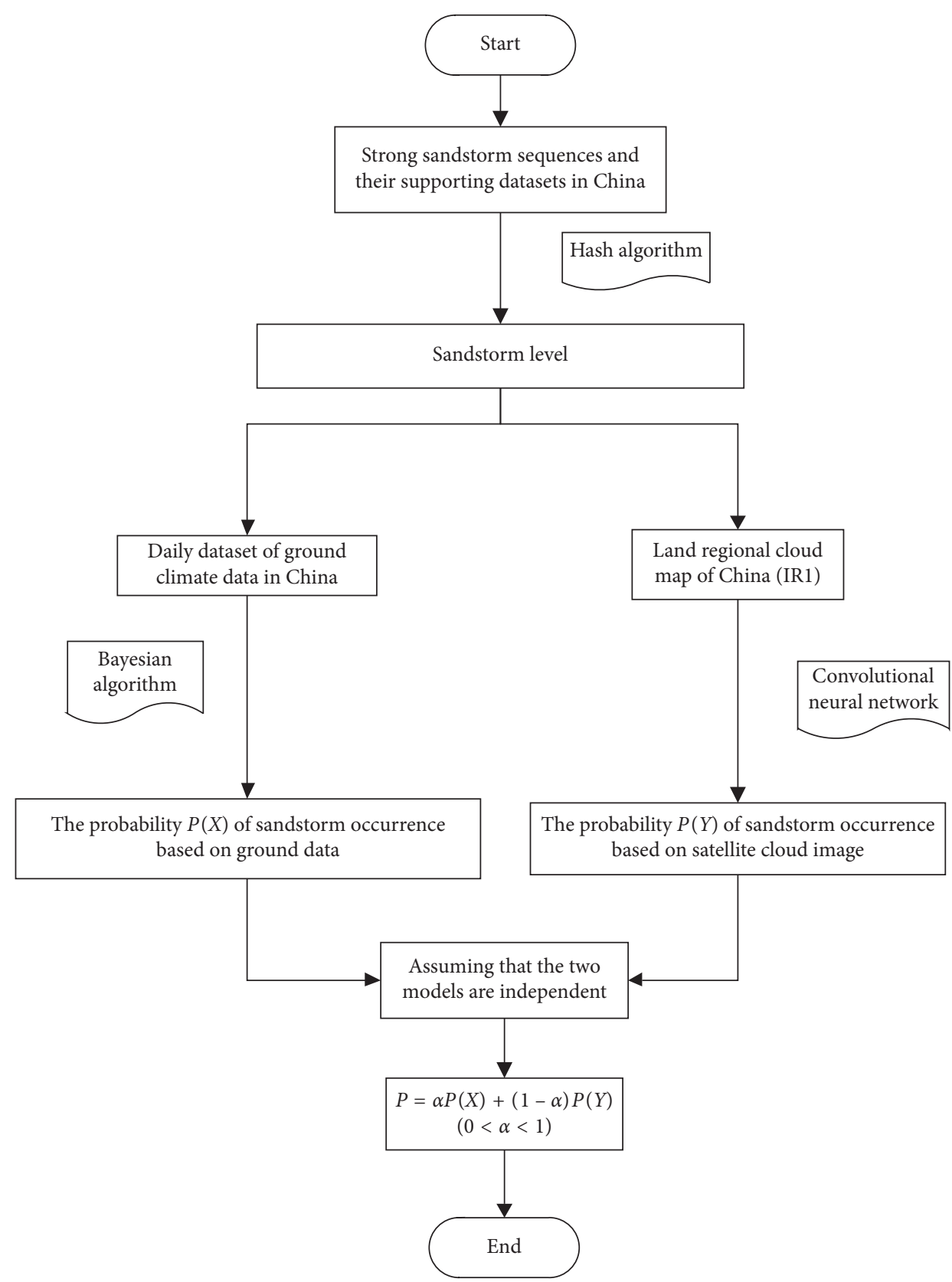

FIGURE 7: The mathematical principle of the INB-CNN classification algorithm.

Assuming that the sandstorm prediction models based on convolutional neural network algorithm and on naive Bayesian algorithm are independent of each other, the optimization problem is transformed into a single-objective optimization problem by weighting. The weight depends on the prediction accuracy of the two models.

The mathematical principle of INB-CNN classification algorithm is described.

In this paper, the intensity of sandstorm occurrence is divided into five grades $\{1,2,3,4,5\}$.

The dataset in Table 1 is used to train and test the sandstorm prediction model based on the naive Bayesian algorithm, and the dataset in Table 3 is used to train and test the sandstorm prediction model based on the convolutional neural network algorithm. Finally, the prediction accuracy of the two models can be analyzed, and the parameter $\alpha$ is determined.

According to the standard of single-station strong sandstorm, the sandstorm and its supporting datasets are used to analyze the sandstorm grade. Then, according to the start and end time of the strong sandstorm, the hash algorithm is used to discretize the sandstorm grade into 30 minutes. The principle of the hash function in this paper is that $y=h$ (key), where key is 30 minutes and $y$ corresponds to the grade of sandstorm occurrence (Algorithm 1).

Taking time as dimension, the test dataset of prediction model based on the INB-CNN classification algorithm is 


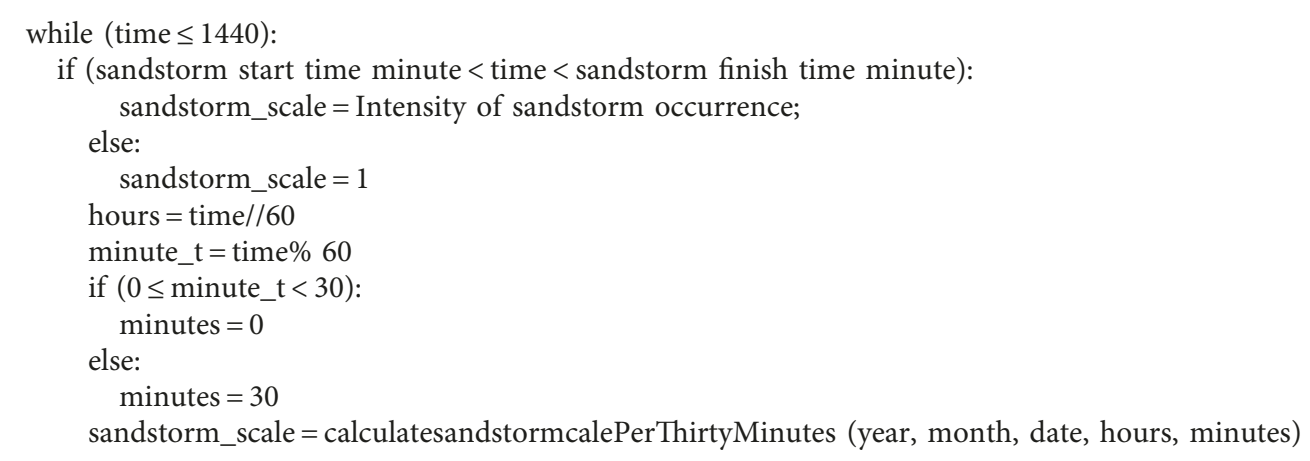

Algorithm 1: Pseudocode for hash function implementation.

obtained, which is integrated with the ground climate data and satellite cloud image data.

Using the test set of the prediction model based on the INB-CNN classification algorithm, the sandstorm prediction model based on the naive Bayesian algorithm was tested to obtain the probability of occurrence of each grade: $P\left(x_{1}\right) P\left(x_{2}\right), P\left(x_{3}\right), P\left(x_{4}\right), P\left(x_{5}\right)$.

Using the test set of the prediction model based on the INB-CNN classification algorithm, the sandstorm prediction model based on the convolutional neural network algorithm was tested to obtain the probability of occurrence of each grade: $P\left(y_{1}\right), P\left(y_{2}\right), P\left(y_{3}\right), P\left(y_{4}\right), P\left(y_{5}\right)$.

The sandstorm prediction model based on the INB-CNN classification algorithm was tested to obtain the probability of occurrence of each grade: $P\left(z_{1}\right), P\left(z_{2}\right), P\left(z_{3}\right), P\left(z_{4}\right), P$ $\left(z_{5}\right), P\left(z_{\mathrm{i}}\right)=\alpha P\left(x_{\mathrm{i}}\right)+(1-\alpha) P\left(y_{\mathrm{i}}\right)$.

\subsection{Training the Prediction Model Based on INB-CNN} Classification Algorithm. The sandstorm prediction model based on the INB-CNN classification algorithm uses three datasets: "China's Series of Strong Dust Storms and Its Supporting Dataset," "Daily Dataset of China's Ground Climate Data," and "China Land Regional Cloud Map (IR1)." The training process of the prediction model based on the INB-CNN classification algorithm is shown in Figure 8 .

Step 1: preprocess the dataset.

Step 2: establish the sandstorm prediction model with naive Bayesian algorithm based on "China Strong Dust Storm Sequence and Its Supporting Dataset" and "Daily Dataset of China's Ground Climate Data.”

Step 3: establish the sandstorm prediction model with convolutional neural network algorithm based on "China Strong Dust Storm Sequence and Its Supporting Dataset" and "China Land Regional Cloud Map (IR1)."

Step 4: the test data are used to test the sandstorm prediction model based on naive Bayesian algorithm and the sandstorm prediction model based on convolutional neural network algorithm, respectively. The predicted probabilities are $P(X)$ and $P(Y)$.
Step 5: based on $P=\alpha P(X)+(1-\alpha) P(Y)(0<\alpha<1)$, the probability of sandstorm occurrence of the sandstorm prediction model based on the INB-CNN classification algorithm is calculated The parameter $\alpha$ is determined by the accuracy of the two models.

Step 6: analyze the experimental results.

5.3. Establishing Sandstorm Prediction Model Based on INBCNN Classification Algorithm. The INB-CNN classification algorithm is used to establish the sandstorm prediction model and study the occurrence law of sandstorms. The sandstorm prediction model based on the INB-CNN classification algorithm is established. The process of establishing the model is shown in Figure 9.

Step 1: take the test set of infrared satellite cloud image as the research object, test the sandstorm prediction model based on convolutional neural network algorithm, and calculate the prediction accuracy as $x$.

Step 2: take the test set of the ground climate data of sandstorm as the research object, test the sandstorm prediction model based on the naive Bayesian algorithm, and calculate the prediction probability as $y$.

Step 3: analyze $x$ and $y$ and calculate the weight factor $\alpha$.

Step 4: in order to find the most likely area of sandstorm in Inner Mongolia every day, the K-means algorithm is used to cluster the test data. Taking the test set as the research object, the dataset of each day in the test data is clustered and the average values of different attributes in each category are calculated. According to the meteorological characteristics of air pressure rise and wind speed increase when sandstorms occur, the most likely area for sandstorm is found. Finally, the data are used to test the sandstorm prediction model based on the naive Bayesian algorithm, and the accuracy of prediction is analyzed.

Step 5: taking the test set of the sandstorm prediction model based on the INB-CNN classification algorithm as the research object, the sandstorm prediction model based on the INB-CNN classification algorithm was tested, and the accuracy of prediction was analyzed. 


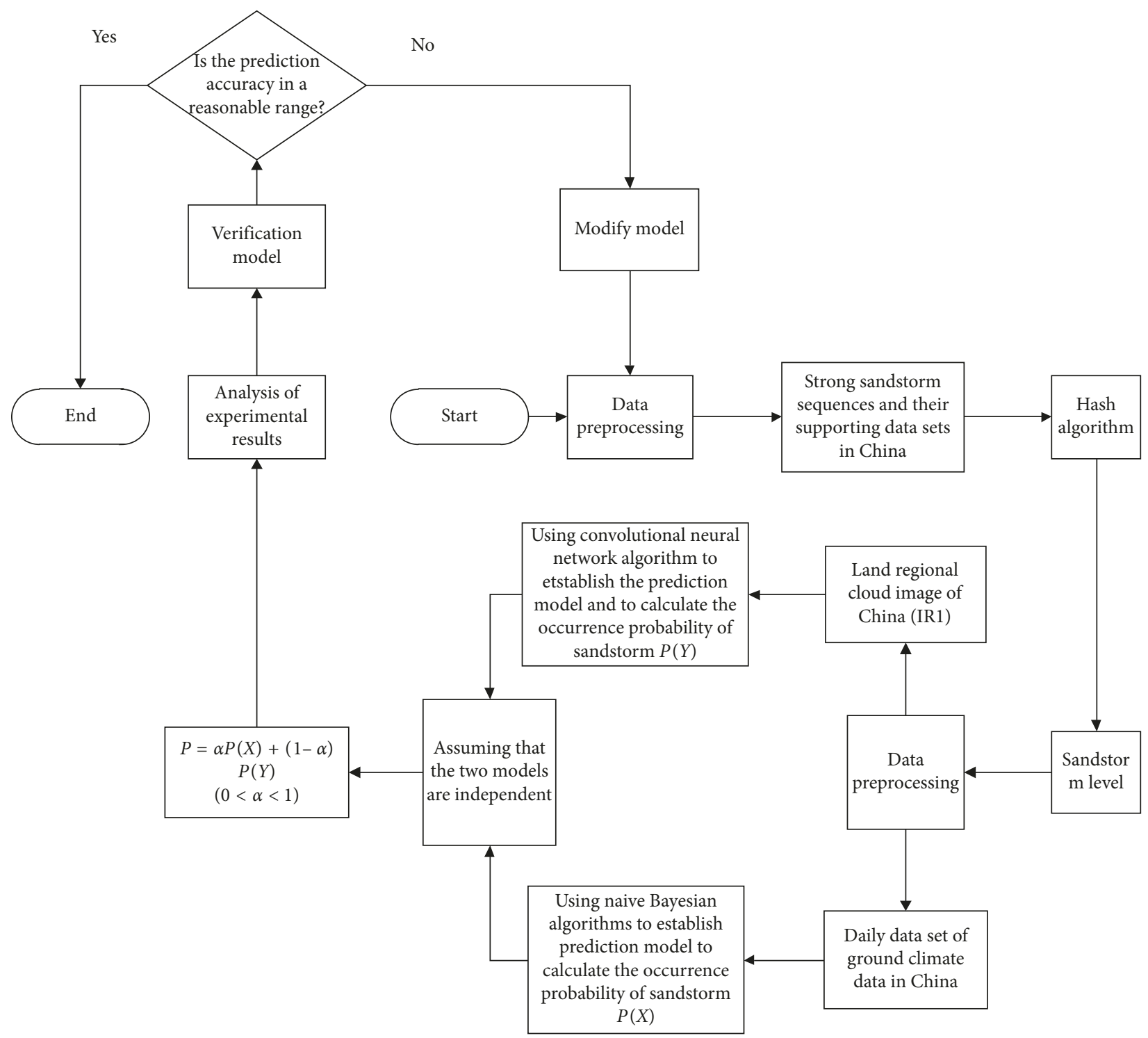

FIgURE 8: Training process of naive Bayesian-CNN classification algorithm.

5.4. Testing Sandstorm Prediction Model Based on INB-CNN Classification Algorithm. After establishing the sandstorm prediction model based on the INB-CNN classification algorithm, it is necessary to test the model. The specific process is as follows:

Step 1: enter the labeled test set data.

Step 2: output the predicted value.

Step 3: compare the predicted value with the actual value and calculate the prediction accuracy.

Step 4: set the reasonable range of the predicted accurate value according to the empirical value.

Step 5: analyze the experimental results.

\section{Results and Discussion}

6.1. Experimental Platform. The experimental environment includes the configuration of system environment and software environment, as shown in Table 4.
6.2. Preparation of Experimental Data. Test dataset of the sandstorm prediction model based on the INB-CNN classification algorithm is shown in Table 5.

6.3. Network Parameter Setting. The solver.prototxt file is used to set the network hyperparameter and control the network operation. The main parameters are configured as shown in Table 6.

6.4. Network Topology. The total number of infrared satellite cloud datasets used is between 20,000 and 30,000, and the number is relatively small. In order to reduce the time of training convolutional neural network and improve the generalization ability of the neural network, the parameter migration in transfer learning is used to initialize the network and train the convolutional neural network. In this process, the model parameters provided by Caffe team are used to initialize the network and fine-tune the model. The 


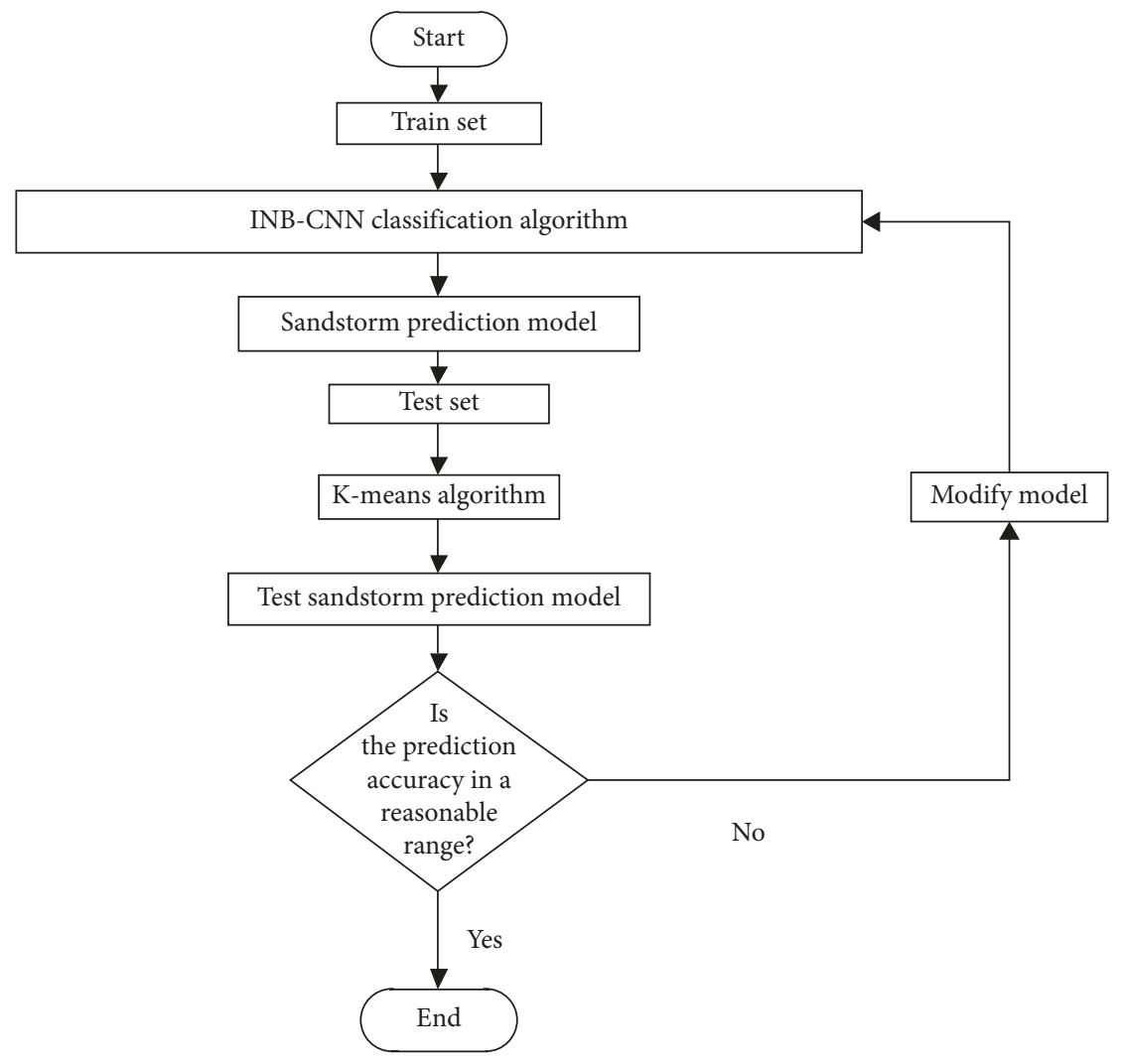

FIGURE 9: Establishment process of sandstorm prediction model based on INB-CNN classification algorithm.

TABle 4: Experimental environment configuration.

\begin{tabular}{lc}
\hline System configuration & Details \\
\hline Operating system & Ubuntu 18.04 LTS \\
Graphic processor unit & NVIDIA GeForce 940MX \\
Memory space & 8.0 GB \\
\hline
\end{tabular}

TABle 5: Test dataset.

\begin{tabular}{cccccccc}
\hline Dataset & 1 & 2 & 3 & 4 & 5 & Total & Unit \\
\hline Test set & 3533 & 24 & 3 & 1 & 4 & 3565 & Line \\
\hline
\end{tabular}

TABLE 6: Convolutional neural network parameter setting.

\begin{tabular}{lc}
\hline Parameter name & Parameter setting \\
\hline test_iter & 100 \\
test_interval & 1000 \\
base_lr & 0.001 \\
lr_policy & "inv" \\
Gamma & 0.1 \\
Display & 20 \\
max_iter & 50000 \\
Momentum & 0.9 \\
weight_decay & 0.0005 \\
Snapshot & 10000 \\
solver_mode & $\mathrm{CPU}$ \\
\hline
\end{tabular}

whole training process of the deep learning model is a process of updating the initialization parameters to the optimal parameters. We used deep learning framework Caffe to implement the convolutional neural network algorithm, processed satellite cloud images, and established the sandstorm prediction model. The network is mainly composed of convolution layer, pooling layer, local response normalization layer, and full connection layer. By normalizing the local input region, the local response normalization layer can effectively improve the accuracy of the network. The activation function used in the network is ReLu. Compared with Sigmoid and Tanh, the ReLu activation function can effectively alleviate the problem of gradient disappearance during network training. The topological structure of convolutional neural network used in this paper is shown in Figure 10.

6.5. Accuracy Curve and Loss Curve. The learning strategy of convolutional neural network used is inv. The convolutional neural network based on inv learning strategy is used to train the neural network. The test interval is 1000 times, and the maximum iteration number is 10000 times. During the training process, the accuracy curve and loss curve of the network is shown in Figure 11.

6.6. Experimental Results and Analysis. We divided the sandstorm grade into five grades: 1, 2, 3, 4, and 5. The grade of 1 represents no sandstorm, and the grades of 1, 2, 3, and 4 


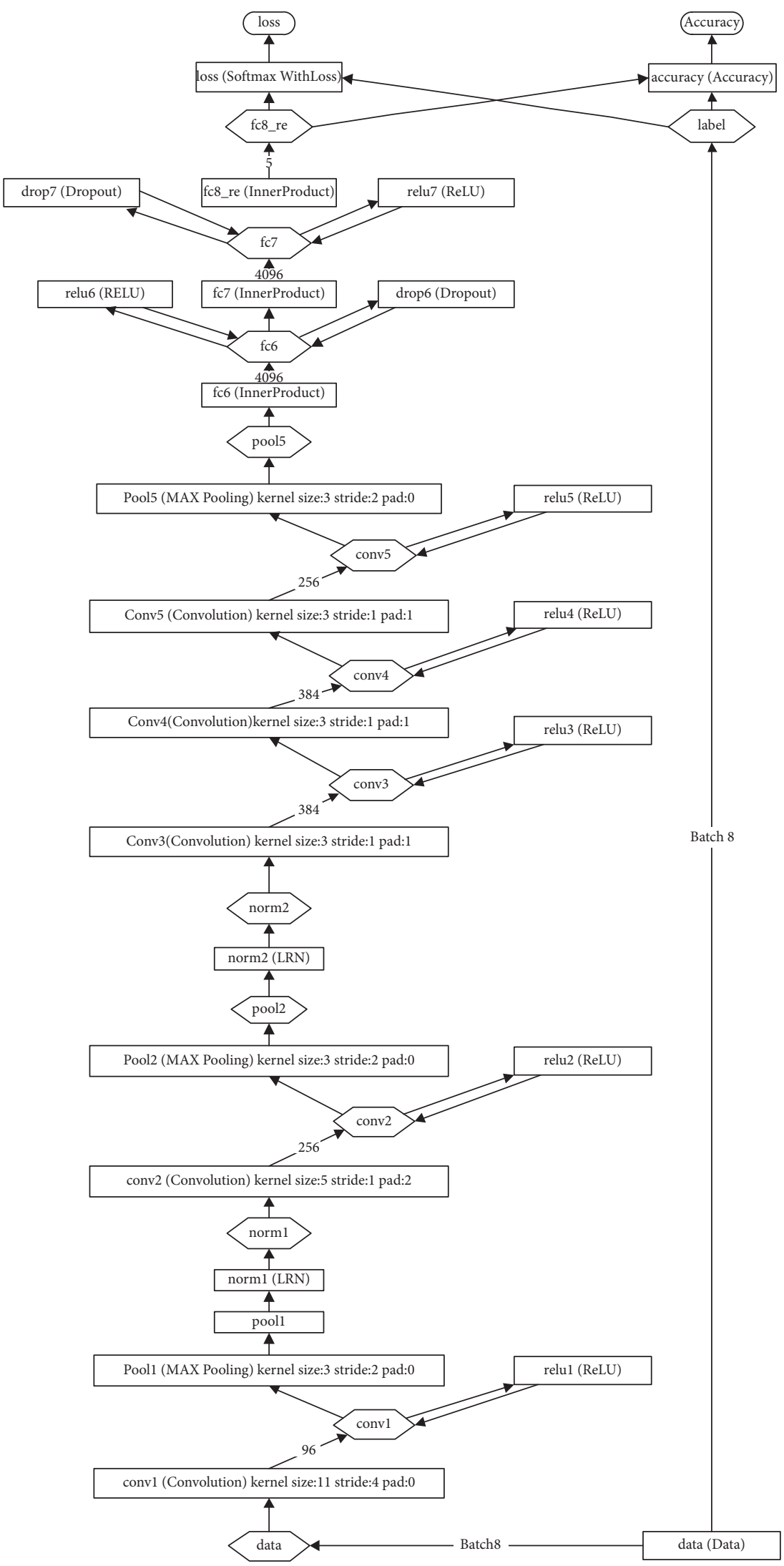

Figure 10: Convolutional neural network topology. 


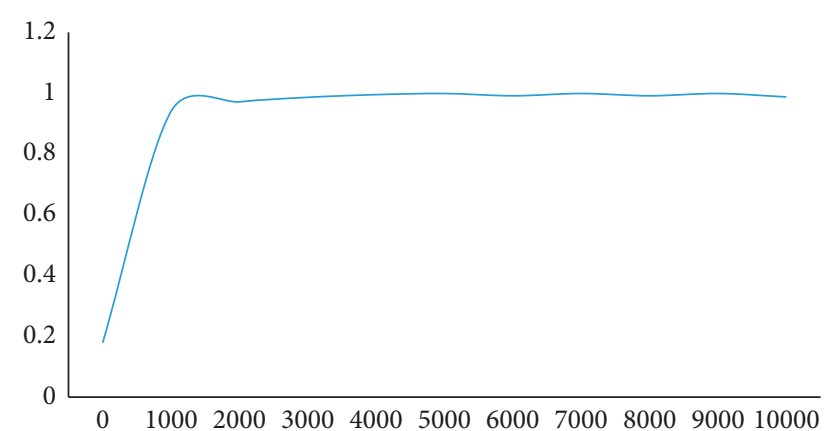

(a)

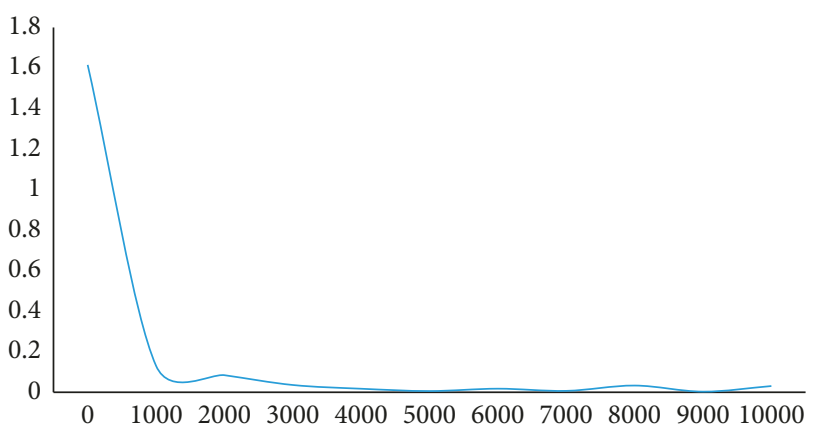

(b)

Figure 11: Accuracy curve and loss curve in inv learning strategy. (a) Accuracy curve. (b) Loss curve.

represent sandstorm. The overall prediction accuracy is the prediction accuracy of the five grades of sandstorm with the research grades of $1,2,3,4$, and 5 ; the accuracy of no sandstorm is the prediction accuracy with the 0 grade; and the accuracy of sandstorm is the prediction accuracy with the grades of $1,2,3$, and 4 .

Taking test set of ground climate data as the research object, the sandstorm prediction model based on naive Bayesian algorithm is tested. Taking the test set of infrared satellite cloud image data as the research object, the sandstorm prediction model based on convolutional neural network algorithm is tested. The experimental results are listed in Table 7.

Taking the test set of ground climate data as the research object, the sandstorm prediction model based on the naive Bayesian algorithm was tested, and the prediction accuracy was 0.531 . Taking the test set of infrared satellite cloud image data as the research object, the sandstorm prediction model based on convolutional neural network algorithm was tested, and the prediction accuracy was 0.833 . Through many experiments, it was found that according to the normalization rule, when the parameter $\alpha$ is set to 0.3 , the accuracy of the sandstorm prediction model based on the INB-CNN classification algorithm is highest.

Taking the test set of the sandstorm prediction model based on the INB-CNN classification algorithm as the research object, the sandstorm prediction model based on the INB-CNN classification algorithm was tested. The experimental results are listed in Table 8 .
TABLE 7: Experimental results.

\begin{tabular}{lcc}
\hline $\begin{array}{l}\text { Prediction types of } \\
\text { sandstorm }\end{array}$ & $\begin{array}{c}\text { Naive Bayesian } \\
\text { algorithm }\end{array}$ & $\begin{array}{c}\text { Convolutional neural } \\
\text { network algorithm }\end{array}$ \\
\hline $\begin{array}{l}\text { Overall prediction } \\
\text { accuracy }\end{array}$ & 0.522 & 0.841 \\
$\begin{array}{l}\text { Prediction accuracy of } \\
\text { no sandstorm }\end{array}$ & 0.456 & 0.873 \\
$\begin{array}{l}\text { Prediction accuracy of } \\
\text { sandstorm }\end{array}$ & 0.531 & 0.833 \\
\hline
\end{tabular}

TABLE 8: Experimental results.

\begin{tabular}{lccc}
\hline $\begin{array}{l}\text { Prediction types } \\
\text { of sandstorm }\end{array}$ & $\begin{array}{c}\text { Naive } \\
\text { Bayesian } \\
\text { algorithm }\end{array}$ & $\begin{array}{c}\text { Convolutional } \\
\text { neural network } \\
\text { algorithm }\end{array}$ & $\begin{array}{c}\text { Improved } \\
\text { algorithm }\end{array}$ \\
\hline $\begin{array}{l}\text { Overall } \\
\text { prediction }\end{array}$ & 0.769 & 0.883 & 0.886 \\
$\begin{array}{l}\text { accuracy } \\
\begin{array}{l}\text { Prediction } \\
\text { accuracy of no }\end{array}\end{array}$ & 0.770 & 0.879 & 0.889 \\
$\begin{array}{l}\text { sandstorm } \\
\text { Prediction } \\
\text { accuracy of }\end{array}$ & 0.312 & 0.375 & 0.406 \\
sandstorm & & & \\
\hline
\end{tabular}

The experimental results show that the accuracy of the model based on the INB-CNN classification algorithm is higher than that of the two models based on naive Bayesian algorithm and convolutional neural network algorithm.

\section{Conclusions}

Sandstorm is an extreme natural disaster. The occurrence of sandstorm will have a serious impact on local economic development and people's lives. Therefore, with the help of advanced intelligent calculation and data mining methods, the analysis of sandstorm meteorological data can help us understand the relevant meteorological laws of sandstorm occurrence in different areas, and it is an effective means to study the occurrence law of sandstorms.

The sandstorm prediction model based on convolutional neural network algorithm takes into account the influence of atmospheric motion; the sandstorm prediction model based on naive Bayesian algorithm takes into account the influence of ground factor. But, both the models consider only a single factor. To solve this problem, the naive Bayesian-CNN classification algorithm is proposed, which takes into account the influence of ground factors and atmospheric motion factors on the occurrence of sandstorm at the same time. Experimental results showed that the prediction accuracy of the sandstorm prediction model based on naive Bayesian-CNN classification algorithm is higher than that of others.

\section{Data Availability}

In this paper, three datasets are used, namely, "China Strong Dust Storm Sequence and Its Supporting Dataset" (data.cma. 
cn/data/detail/dataCode/SURF_CLI_CHN_WEP DAY_DSS/ keywords/China's Series of Strong Dust Storms and Its Supporting Data Set.html), "China Surface Climate Data Daily Dataset” (http://data.cma.cn/data/detail/dataCode/SURF_CLI_CHN_MUL_DAY_V3.0/keywor_ds/Daily Data Set of China's Ground Climate Data.html), and "China Land Regional Cloud Map (IR1)” (http://data.cma.cn/data/detail/ dataCode/SK.0137.001/keywords/China Land Regional Cloud Map (IR1).html). The three datasets were all obtained from China Meteorological Data Network (http:// data.cma.cn/site/index.html), a national platform for basic scientific and technological conditions.

\section{Conflicts of Interest}

There are no conflicts of interest regarding the publication of this paper.

\section{Acknowledgments}

The authors thank their mentor Ren Qing-dao-er-ji for his guidance and help and also thank Inner Mongolia University of Technology for its good research environment. This work was financially supported by the Natural Science Foundation of Inner Mongolia (2018MS06021 and 2016MS0605).

\section{References}

[1] Q.-C. Zeng, C.-H. Dong, G.-B. Peng, Si-X. Zhao, Z.-Y. Fang, and M.-Y. Jiao, "Sandstorm related disaters," Climatic and Enviromental Research, vol. 12, no. 3, pp. 225-226, 2007.

[2] C. Chang-qing, J. Jin-rong, and C. Xue-bin, "Application of grid nesting techniques on cuace-dust," in Proceedings of the 2012 international conference on industrial control and electronics engineering, pp. 962-964, Xi'an, China, August 2012.

[3] K. Wei, T. Zhang, and B. He, "Detection of sand and dust storms from MERIS image using FE-otsu alogrithm," in Proceedings of the 2008 2nd International Conference on Bioinformatics and Biomedical Engineering, pp. 3852-3855, Shanghai, China, May 2008.

[4] H. Han, N. Guo, D. Cai, and J. Wang, "FY-2D retrieved surface temperature change as a predictor for sandstorm forecasting over Northwest China," in Proceedings of the 2011 IEEE International Geoscience and Remote Sensing Symposium, pp. 3261-3264, Vancouver, BC, Canada, July 2011.

[5] Z. Guoping, L. Jiyuan, and Z. Zenxiang, "Remote sensing investigation of the main sand-supplying areas of dust storm hitting northern China," in Proceedings of the IGARSS 2001. Scanning the Present and Resolving the Future. Proceedings. IEEE 2001 International Geoscience and Remote Sensing Symposium (Cat. No.01CH37217), vol. 5, pp. 2097-2099, Sydney, NSW, Australia, July 2001.

[6] Z. Lu, J. Dai, Y. Yang, and H. Liu, "Research on the forecasting model of sand-dust storm based on the grid field," in Proceedings of the Third International Conference on Natural Computation (ICNC 2007), pp. 348-352, Haikou, China, August 2007.

[7] S.-B. Kim, K. Yumimoto, I. Uno, and Y. Chun, "Dust model intercomparison between ADAM and CFORS/dust for asian dust case in 2007 (march 28-april 3)," Sola, vol. 7A, no. Special_Edition, pp. 25-28, 2011.
[8] H. J. In and S. U. Park, "A simulation of long-range transport of Yellow Sand observed in April 1998 in Korea," Atmospheric Environment, vol. 36, no. 26, pp. 4173-4187, 2002.

[9] M. Akhlaq, T. R. Sheltami, and E. M. Shakshuki, "Developing a hybrid system for sand and dust storm detection using satellite imaging and WSNs," in Proceedings of the International Conference on Information Integration and WebBased Applications \& Services, pp. 9-15, ACM, Salzburg, Austria, December 2012.

[10] C. Pérez, J. M. Baldasano, P. Jiménez-Guerrero et al., “Dust modelling and forecasting in the barcelona supercomputing center: activities and developments," IOP Conference Series: Earth and Environmental Science, vol. 7, Article ID 012013, 2009.

[11] M. Gholoum, D. Bruce, and S. Alhazeem, "A new image classification approach for mapping coral density in State of Kuwait using high spatial resolution satellite images," International Journal of Remote Sensing, vol. 40, no. 12, pp. 4787-4816, 2019.

[12] L. Liu, Y. Zhao, P. Du et al., "SPIE proceedings (SPIE geoinformatics 2008 and joint conference on GIS and built environment: classification of remote sensing images-Guangzhou, China (saturday 28 june 2008)) geoinformatics 2008 and joint conference on GIS and built environment: classification of remote sensing images-incorporating spectral, texture, and shape information for high spatial resolution satellite imagery classification," Proceedings of SPIE-The International Society for Optical Engineering, vol. 7147, Article ID 714719, 2008.

[13] M. L. Campagnolo and M. Caetano, "Expert maps: an alternative for integrating expert knowledge in satellite imagery classification," in Proceedings of the Geoscience \& Remote Sensing, Igarss 97 Remote Sensing-a Scientific Vision for Sustainable Development, IEEE International, Singapore, August 1997.

[14] M. Nguyen, J. Block, D. Crawl et al., "Land cover classification at the wildland urban interface using high-resolution satellite imagery and deep learning," in Proceedings of the 2018 IEEE International Conference on Big Data (Big Data), vol. 16321638, Seattle, WA, USA, December 2018.

[15] B. Zhang, A. Tsunekawa, and M. Tsubo, "Contributions of sandy lands and stony deserts to long-distance dust emission in China and Mongolia during 2000-2006," Global and Planetary Change, vol. 60, no. 3-4, pp. 487-504, 2008. 

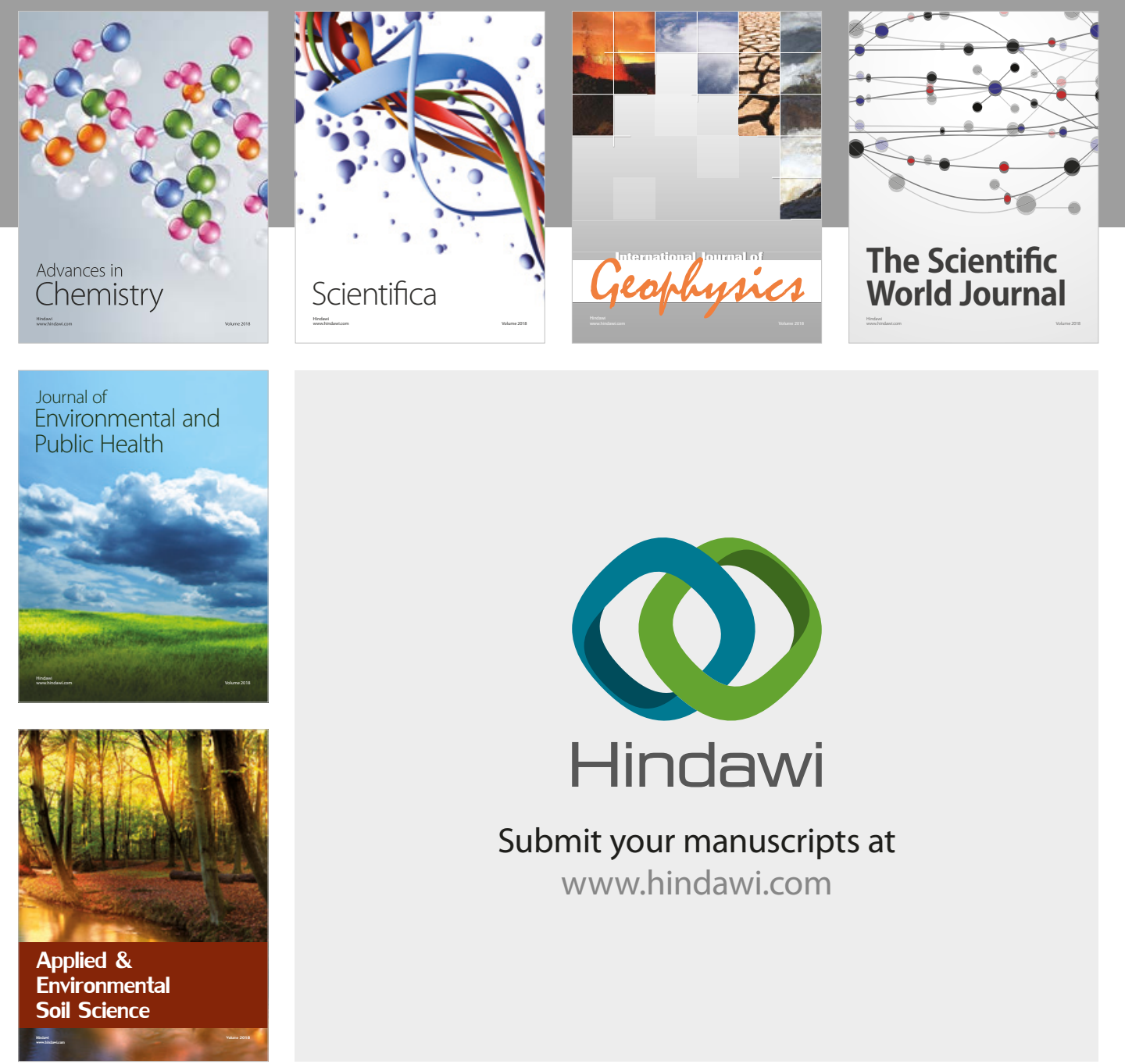

The Scientific

\section{World Journal}
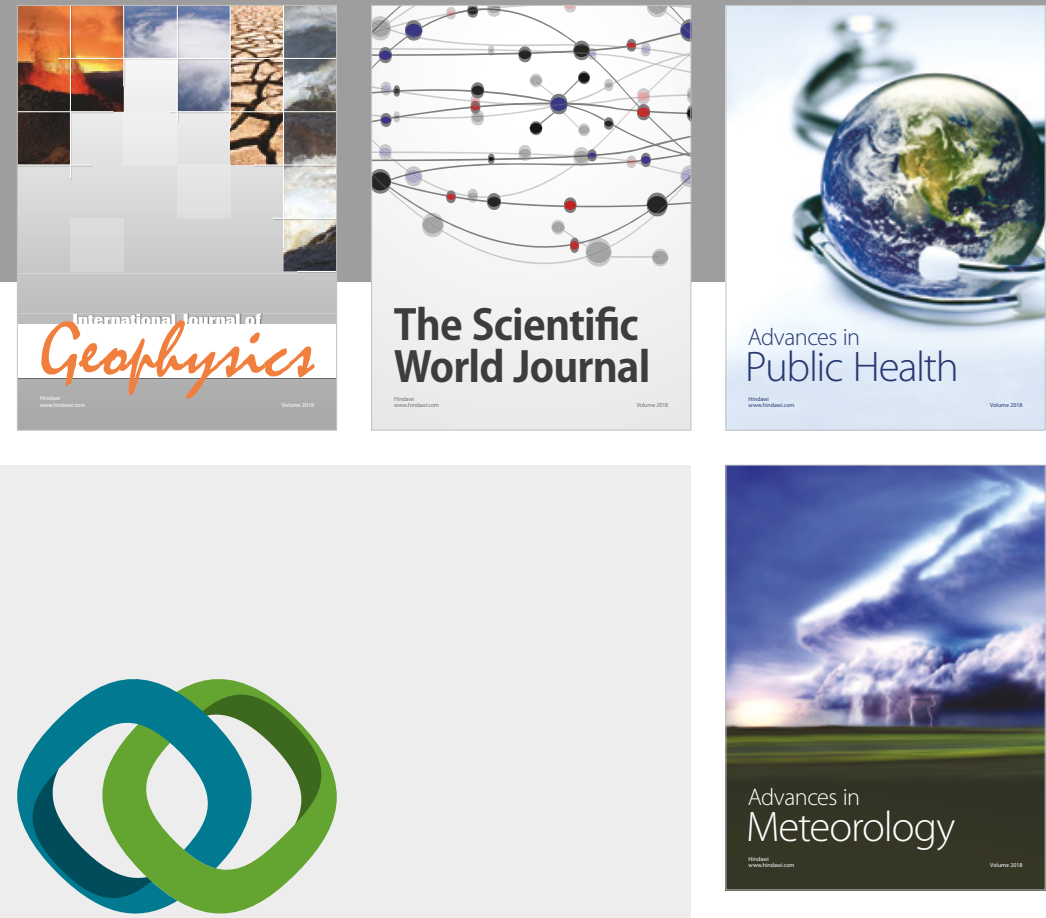

Advan

Public Health

\section{Hindawi}

Submit your manuscripts at

www.hindawi.com
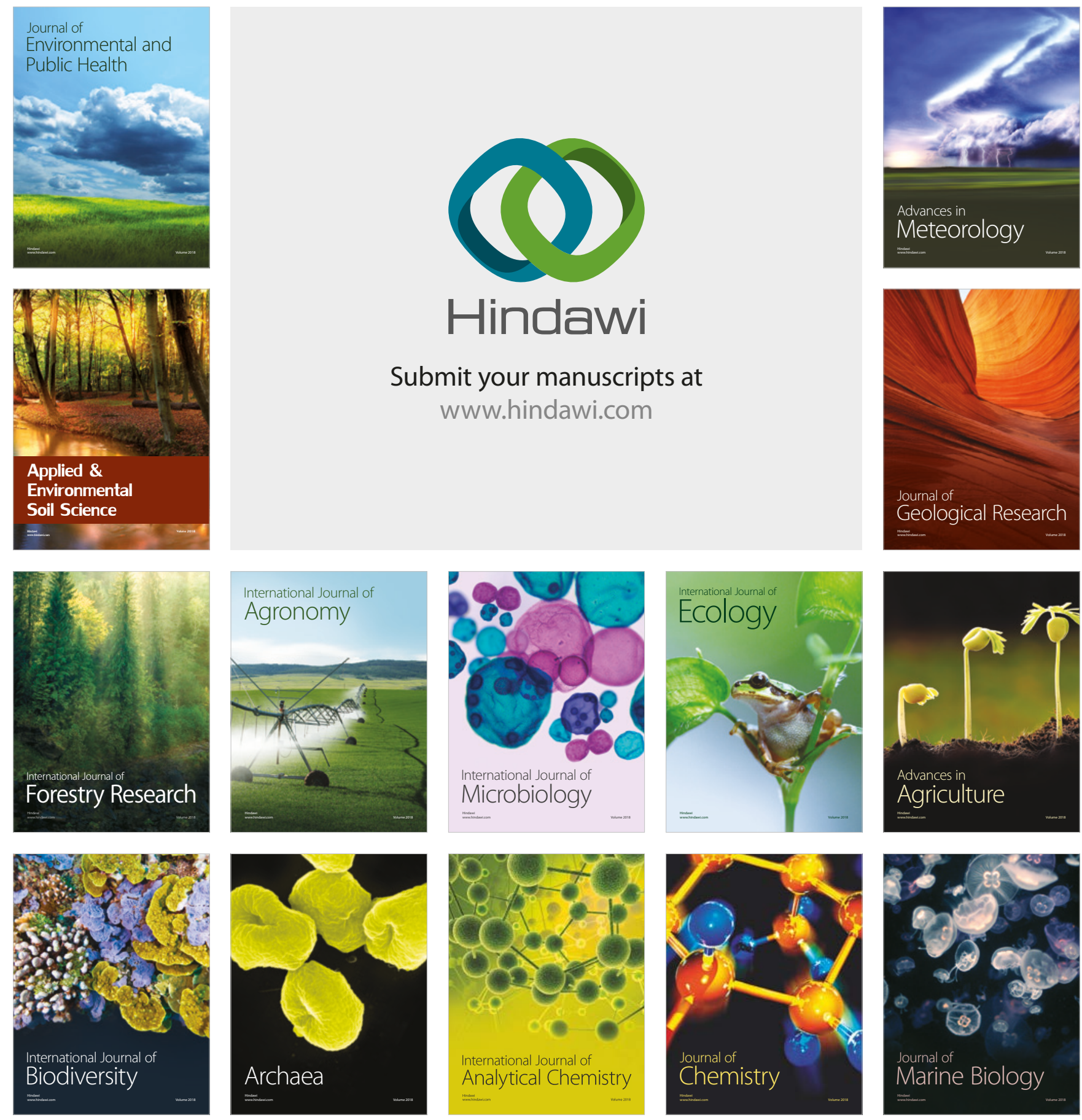